\title{
COMPORTAMENTO DO MERCÚRIO EM PERFIS DE SOLOS DO SÍTIO ILHA DE TERRA-CAXIUANÃ, PARÁ
}

Robson da Silva Gurjão, Vanda Porpino Lemos*, Marcondes Lima da Costa, Heronides Adonias Dantas Filho, Kelly das Graças Fernandes Dantas e Wivian Thais dos Santos Lima

Instituto de Ciências Exatas e Naturais, Universidade Federal do Pará, 66075-780 Belém - PA, Brasil

Dirse Clara Kern

Museu Paraense Emílio Goeldi, Belém - PA, Brasil

Recebido em 29/4/09; aceito em 29/10/09; publicado na web em 24/3/10

\begin{abstract}
BEHAVIOR OF MERCURY IN SOIL PROFILES FROM SÍTIO ILHA DE TERRA-CAXIUANÃ, PARÁ. Behavior of mercury in soil profiles with archaeological black earth (ABE) and surroundings area (SA) from Sítio Ilha de Terra, Caxiuanã, can provide information on anthropogenic activity of the Amazonian habitat. The samples of ABE and SA soil profiles were submitted to mineralogical chemical (total and sequential) analysis. The data show that the $\mathrm{Hg}$ occurs mainly in goethite and kaolinite in the two soil profiles. The highest concentrations of $\mathrm{Hg}$ and Fe are observed in the SA profile. These results indicate that the prehistoric human occupation contributed to the decrease of the concentration of $\mathrm{Hg}$ in soil $\mathrm{ABE}$ from Caxiuanã.
\end{abstract}

Keywords: archaeological black earth; mercury; Caxiuanã.

\section{INTRODUÇÃO}

A concentração de mercúrio na crosta terrestre superior é 56 ng $\mathrm{g}^{-1}$ e ocorre na biosfera sob as formas inorgânicas e orgânicas. ${ }^{1}$ As formas inorgânicas são o $\mathrm{Hg}$ elementar, íon mercúrio dimérico e íon mercúrico; as principais formas orgânicas são o metilmercúrio $\left(\mathrm{CH}_{3} \mathrm{Hg}^{+}\right)$e dimetilmercúrio [ $\left.\left(\mathrm{CH}_{3}\right)_{2} \mathrm{Hg}\right]$. O metilmercúrio é a espécie mais tóxica do mercúrio e sofre bioacumulação através de vários processos, tais como ingestão de alimentos e ação antrópica ao longo da cadeia alimentar aquática. ${ }^{2}$ Durante longo período foram consideradas como as principais fontes de contaminação por $\mathrm{Hg}$ na Amazônia as produzidas pelas atividades de garimpo, pelos mecanismos de dispersão e a acumulação nos solos, sedimentos e águas. ${ }^{3,4}$ Entretanto, tem sido demonstrado nas últimas décadas que as fontes emissoras mais importantes são desmatamento, queimadas, destruição das raízes dos solos ${ }^{5}$ o $\mathrm{Hg}$ associado aos solos lateríticos..$^{6-8}$

As queimadas são responsáveis pela mobilização do $\mathrm{Hg}$ contido na biomassa, redistribuição do Hg na atmosfera na forma de vapor ou ligado ao material particulado e precipitação nos sistemas aquáticos, participando nos processos de bioacumulação ao longo da cadeia trófica, sendo consideradas atualmente a principal fonte de emissão de $\mathrm{Hg}$ atmosférico na Amazônia. A destruição dos sistemas radiculares, realizada nas áreas de queimadas que são preparadas para o cultivo de plantas e pastos, conduz à perda da estrutura dos solos que ficam mais expostos à ação das chuvas torrenciais típicas da região Amazônica, propiciando assim que os solos exportem para o sistema aquático grandes quantidades de material particulado contendo $\mathrm{Hg} .{ }^{5,6}$

Os defensores da contribuição do $\mathrm{Hg}$ associado aos solos lateríticos apontam os solos da Amazônia como grandes reservatórios naturais de $\mathrm{Hg}$ associado aos óxidos e hidróxidos de ferro que, ao sofrerem transformações a partir de processos naturais ou antrópicos, disponibilizam o $\mathrm{Hg}$ para os sistemas aquáticos. ${ }^{9}$

As fontes potenciais de emissão de $\mathrm{Hg}$ referidas acima e sua redistribuição no ambiente amazônico são reconhecidas pela comunidade científica, mas ainda não há um consenso sobre a representatividade dos processos envolvidos como determinantes do comprometimento nos ecossistemas aquáticos e as justificativas para elevadas concentrações

*e-mail: vplemos@ufpa.br de Hg observadas em solos e sedimentos. ${ }^{10,11}$ Estudos realizados sobre estas questões identificam duas vertentes que tentam explicar a origem do $\mathrm{Hg}$ e sua disseminação em outros compartimentos. A primeira, com argumentação em favor da origem antrópica, representada pelas emissões de $\mathrm{Hg}$ metálico ligado à atividade garimpeira, atualmente em declínio, ${ }^{12,13}$ defende a ideia que o $\mathrm{Hg}$ emitido para a atmosfera e transportado a longa distância seria responsável pela contaminação da Bacia Amazônica. A segunda defende a ideia de que os solos amazônicos atuariam como reservatórios naturais de $\mathrm{Hg}$, mas reconhece a atividade garimpeira como uma fonte importante de $\mathrm{Hg}$ e com área de abrangência restrita às proximidades dos locais de emissão. ${ }^{9}$

Em estudos realizados em uma área de exploração geoquímica, denominada de Águas Claras, na Província Mineral de Carajás-Pará foram obtidos teores médios de $\mathrm{Hg}$ em latossolos na ordem de $196 \mathrm{ng}$ $\mathrm{g}^{-1}$ e em materiais lateríticos na ordem de $329 \mathrm{ng} \mathrm{g}^{-1} \cdot{ }^{14}$ Os resultados deste estudo comparados com os obtidos em áreas sem registros de atividades antrópicas de $\mathrm{Hg}$ indicaram que a ocorrência do $\mathrm{Hg}$ é natural de ambiente supergênico, mas com teores mais elevados do que os admitidos como background na região Amazônica. Em outros estudos sobre avaliações das concentrações de $\mathrm{Hg}$, foram observados: em amostras de solos coletadas ao longo de rodovias no Estado do Acre, que o acúmulo natural de $\mathrm{Hg}$ está relacionado com os processos de formação dos solos, levando-se em consideração os teores elevados de $\mathrm{Hg}$ na rocha original da Formação Solimões e que durante o intemperismo laterítico ou desenvolvimento dos solos sob condições tropicais o $\mathrm{Hg}$ tende a ser remobilizado e enriquecido na parte superior dos perfis, geralmente associado aos óxidos e hidróxidos de férreo; ${ }^{11}$ em amostras de solos de áreas de garimpos da região de Tapajós teores de $\mathrm{Hg}$ em perfis de alteração (teores correspondentes aos horizontes da base ao topo dos perfis) nos seguintes intervalos: $18-117 \mathrm{ng} \mathrm{g}^{-1}$ no saprólito, 77-123 $\mathrm{ng} \mathrm{g}^{-1}$ na zona de transição, 88-385 $\mathrm{ng} \mathrm{g}^{-1}$ na crosta ferruginosa, 100-222 $\mathrm{ng} \mathrm{g}^{-1}$ nos latossolos. Estes dados indicaram que a origem do $\mathrm{Hg}$ é devida não somente à pedogênese, mas também ao aporte externo provenientes das minas de Au e Ag, exploradas na América do Sul desde os tempos coloniais, e pela garimpagem praticada na região nos últimos 30 anos. ${ }^{12,13}$

Neste estudo as avaliações das concentrações de $\mathrm{Hg}$ foram realizadas em uma área de conservação ambiental localizada na região de Caxiuanã. Nesta região o Hg está incluído em um grupo de elementos 
que vem sendo investigado em solos com terra preta arqueológica (TPA) a fim de elucidar hipóteses sobre o comportamento dos antigos habitantes da região de Caxiuanã. ${ }^{15,16} \mathrm{~A}$ região é formada pela baía de Caxiuanã e suas terras vizinhas integrantes dos municípios de Portel e Melgaço e nela está situada a Floresta Nacional de Caxiuanã, administrada pelo IBAMA. Nesta região há uma grande área (aproximadamente 33.000 ha) cedida ao Museu Paraense Emílio Goeldi, denominada Estação Científica Ferreira Penna. ${ }^{17}$ Às margens da baia de Caxiuanã há ocorrências de solos de cor preta, com evidências de grande influência do homem pré-histórico, como fragmentos cerâmicos microgranulares e elevadas concentrações de Ca, Mg, Zn, Mn, P e C. Os solos com estas características foram descobertos em outros locais bem drenados às margens de vários rios da Amazônia e foram denominados Terra Preta de Índio, ${ }^{18}$ Terra Preta Arqueológica ${ }^{15,16}$ ou Terra Preta Amazônica. ${ }^{19}$ No sítio Manduquinha foram observadas associações de elementos que deram indícios das seguintes informações: $\mathrm{P}, \mathrm{Mg}, \mathrm{Ca}, \mathrm{Cu}, \mathrm{Cl}, \mathrm{Mn}, \mathrm{Sr}$ e $\mathrm{Zn}$ - elementos adicionados ao solo; Fe, Na, As, Cd, Co, Cr, F, Ga, Pb e V concentrações modificadas pela atividade humana pré-histórica; $\mathrm{B}, \mathrm{Hg}, \mathrm{Nb}, \mathrm{Sc}$ e Y - elementos que não sofreram alterações significativas em decorrência da ocupação humana pré-histórica. No sítio Manduquinha foram avaliadas as concentrações de $\mathrm{Hg}$ em perfis de solos em áreas com TPA em relação às obtidas em áreas adjacentes $(\mathrm{AD})$ e de outras regiões da Amazônia. ${ }^{14}$ Foi verificado que nas áreas com TPA as concentrações de $\mathrm{Hg}$ se encontram no intervalo 65-105 $\mathrm{ng} \mathrm{g}^{-1}$ e nas AD, em teor médio de $121 \mathrm{ng} \mathrm{g}^{-1}$. Em outros sítios da região de Caxiuanã foram obtidos teores de Hg nas áreas com TPA entre 202-204 ng g-1 e entre 203-230 ng g ${ }^{-1}$ nas áreas AD. ${ }^{20}$ No sentido de dar continuidade ao estudo sobre concentrações de elementos que podem ser modificadas pelas atividades humanas pré-históricas foi avaliada a partição do $\mathrm{Hg}$ em relação aos principais atributos químicos e mineralógicos em dois perfis de solos, um perfil com TPA e outro em área adjacente (perfil AD) em um sítio arqueológico, denominado Ilha de Terra na região de Caxiuanã, município de Melgaço, estado do Pará.

\section{PARTE EXPERIMENTAL}

\section{Materiais e métodos}

Às margens da baía de Caxiuanã há ocorrências de vários sítios arqueológicos, sendo até o presente descobertos 27 sítios distribuídos ao longo das direções norte e sul da baía. O sítio Ilha de Terra está situado no extremo norte da baía, às margens do Furo Camuim. As amostragens neste sítio foram realizadas em dois perfis de solos, um em área com terra preta arqueológica (perfil TPA) e outro em área adjacente (perfil AD), sendo aplicadas as técnicas de amostragens recomendadas pela Sociedade Brasileira de Ciência do Solo. ${ }^{21}$ As cores dos horizontes dos perfis de solos foram caracterizadas com base nos códigos da carta de Munsell. ${ }^{22}$

A fração menor que $2 \mathrm{~mm}$ de diâmetro, após secagem ao ar, denominada terra fina seca ao ar (TFSA), foi utilizada nas análises granulométrica, ${ }^{23}$ mineralógica, química total e na determinação do $\mathrm{pH}$. A análise mineralógica foi efetuada por difração de raios X através de um difratômetro Philips, modelo PW 1050 com sistema de controle PW 3710 e anodo de cobre. $\mathrm{O}$ pH em água e em $\mathrm{KCl}$, a perda ao fogo e a matéria orgânica foram determinados de acordo com os métodos da USDA. ${ }^{24} \mathrm{O}$ pH das amostras em água e em solução de $\mathrm{KCl}$ foi determinado através do método potenciométrico direto, utilizando-se razão sólido:líquido igual a $1 \mathrm{~g}$ : $25 \mathrm{~mL}$ e repouso por 30 min. A diferença entre os valores de $\mathrm{pH}, \mathrm{pH}=\mathrm{pH}_{\mathrm{KCl}}-\mathrm{pH}_{\mathrm{H} 2 \mathrm{O}}$, dá uma estimativa da carga líquida do solo. A perda ao fogo foi determinada a partir da liberação dos constituintes voláteis a $900{ }^{\circ} \mathrm{C}$ e a matéria orgânica pelo método de oxi-redução usando-se o dicromato de potássio como oxidante da matéria orgânica. A partir de solução amostra obtida por fusão alcalina com metaborato de lítio e dissolução do resíduo de fusão em $\mathrm{HCl}$ 1:1 foram determinados: $\mathrm{SiO}_{2}$ por gravimetria; $\mathrm{Al}_{2} \mathrm{O}_{3}$ total por complexometria com EDTA; $\mathrm{Fe}_{2} \mathrm{O}_{3}$ total por espectrofotometria, sob a forma do complexo orto-fenantrolina ferrosa, após redução dos íons $\mathrm{Fe}^{3+}$ (provenientes da goethita) e reação entre os íons $\mathrm{Fe}^{2+}$ e orto-fenantrolina em $\mathrm{pH}=3,76$ e, $\mathrm{TiO}_{2}$ total por espectrofotometria como peróxido complexo. ${ }^{25}$

\section{Análise do mercúrio}

\section{Digestão ácida para determinação da concentração do mercúrio total}

A digestão ácida foi efetuada pelo método de Akagi e Nishimura, ${ }^{26}$ que consiste nas seguintes etapas: tratamento ácido das amostras com $2 \mathrm{~mL}$ de solução de ácido nítrico $\left(\mathrm{HNO}_{3}\right)$ - ácido perclórico $\left(\mathrm{HClO}_{4}\right)$ (1:1) para decompor a matéria orgânica; tratamentos com $5 \mathrm{~mL}$ de ácido sulfúrico concentrado e $1 \mathrm{~mL}$ de $\mathrm{H}_{2} \mathrm{O}$ Milli-Q para transformar os metais presentes em sulfatos; repouso das soluções-amostra por 12 $\mathrm{h}$ e aquecimento posterior em temperatura de $220^{\circ} \mathrm{C}$ durante $20 \mathrm{~min}$, a fim de melhorar a eficiência do processo de digestão e, diluição das soluções-amostra para $50 \mathrm{~mL}$ com água Milli-Q.

\section{Extração sequencial do $\mathrm{Hg}$}

A determinação das concentrações de espécies químicas em diferentes componentes presentes em amostras de solos vem sendo efetuada a partir de métodos de extração sequencial ou seletiva que compreendem 4 a 5 fases sucessivas de tratamentos químicos, envolvendo solubilização preliminar em água (nos casos de sedimentos marinhos e de mangues) e com outros reagentes extratores apropriados à disponibilização de íons trocáveis a partir de fases constituídas de minerais com estruturas favoráveis à capacidade de troca iônica, à adsorção de íons complexos em sítios adequados de óxidos e hidróxidos amorfos e cristalinos de $\mathrm{Fe}$ e $\mathrm{Mn}$, como nos polimorfos do $\mathrm{FeOOH}$ (goethita e lepidocrocita) e do $\mathrm{Fe}_{2} \mathrm{O}_{3}$ (hematita e maghemita), em substâncias húmicas e em outros componentes presentes na fase residual que podem ser minerais pesados (zircão, cromita etc.) e/ou minerais de argila (caulinita, esmectita, vermiculita etc.).

Os métodos de extração sequencial aplicados às análises de metais em solos e sedimentos são geralmente adaptações dos métodos de Tessier, Chao, Miller, Keller e Hall, ${ }^{27-31}$ que se diferenciam especialmente pela seletividade dos extratores utilizados e pelos componentes de interesse presentes na amostra analisada. A característica comum entre esses métodos é não terem alcançado rendimento total na disponibilização das espécies químicas desejadas. Este aspecto tem chamado atenção nos trabalhos envolvendo revisão e avaliação de procedimentos de extrações sequenciais de metais em solos. ${ }^{28,32} \mathrm{Os}$ principais fatores considerados pelo aumento da seletividade desses métodos são seleção dos reagentes extratores, tempo de extração e razão entre o volume de cada extrator e o peso da amostra analisada. Os procedimentos de extração sequencial recomendados por BCR da Comunidade Europeia (atualmente The Standards, Measurement and Testing Programme) e utilizados por Davidson et al., ${ }^{33}$ diferenciam-se dos métodos referidos acima pelo número de estágios envolvidos no processo de extração, que são três, e por não considerarem a extração dos metais adsorvidos nos minerais de argila que se dá a partir da dissolução total desses minerais em mistura ácida $\mathrm{HClO}_{4}: \mathrm{HF}$.

Neste trabalho foram utilizados os procedimentos de Hall et al. ${ }^{31}$ que consideram a extração de metais em minerais de argila e deu-se grande atenção às recomendações da IUPAC sobre o uso inadequado de terminologias durante as avaliações das concentrações obtidas nas distintas fases de extração. ${ }^{34}$ No método de Hall et al.,${ }^{31}$ as fases de maior interesse são assim agrupadas: 1- fase associada aos cátions 
trocáveis, adsorvidos e/ou ligados a carbonatos; 2- fase associada aos oxi-hidróxidos de ferro amorfos; 3 - fase associada aos óxidos de ferro cristalinos; 4- fase associada à matéria orgânica e sulfetos; 5- fase ligada aos silicatos e óxidos residuais. Essas técnicas, com pequenos ajustes, foram aplicadas neste trabalho. Os detalhes dos procedimentos estão resumidos abaixo:

1. O Hg é disponibilizado sob a forma trocável e/ou adsorvido após tratamento de $0,5 \mathrm{~g}$ da amostra com solução de acetato de amônio $1 \mathrm{~mol} \mathrm{~L}{ }^{-1}$, que pode ser substituída por soluções de $\mathrm{CaCl}_{2}$, $\mathrm{MgCl}_{2}$ ou $\mathrm{BaCl}_{2}$.

2. O Hg é liberado junto com a matéria orgânica, mediante trata-

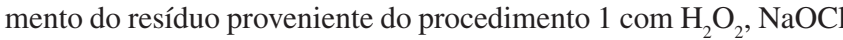
ou $\mathrm{H}_{2} \mathrm{O}_{2}$ mais $\mathrm{HNO}_{3}$ diluído.

3. O Hg disponibilizado na fase dos óxi-hidróxidos de ferro é solubilizado após tratamento do resíduo proveniente do procedimento 2 com solução de $\mathrm{HCl} 0,5 \mathrm{~mol} \mathrm{~L}^{-1}$.

4. $\mathrm{O} \mathrm{Hg}$ presente nos silicatos é disponibilizado através de tratamento do resíduo do procedimento 3 com $\mathrm{HClO}_{4}$ : $\mathrm{HF}$ (1: 5), evaporação e tratamento adicional do resíduo com $\mathrm{HNO}_{3}$ diluído.

\section{Análise do Hg: total e sequencial}

As concentrações de $\mathrm{Hg}$, total e nas distintas fases obtidas a partir de extração sequencial, foram determinadas por espectrometria de absorção atômica com sistema de geração de vapor frio de $\mathrm{Hg}(\mathrm{CV}$ AAS). Nesta técnica os íons $\mathrm{Hg}^{2+}$ são reduzidos sob a forma elementar e os vapores de $\mathrm{Hg}$ produzidos são carreados por um gás até o caminho óptico do espectrômetro. Esta técnica, segundo Micaroni et al., ${ }^{35}$ é a mais comum na análise do Hg. As determinações de Hg nas soluçõesamostra foram feitas usando-se um sistema de geração de vapor frio acoplado a um espectrômetro de absorção atômica, HG-3500, K.K. Sanso. As soluções-amostra foram processadas com base no procedimento adaptado por Akagi et al. ${ }^{36}$ que envolve as seguintes etapas: redução do $\mathrm{Hg}^{2+}$ com solução ácida de $\mathrm{SnCl}_{2}$; transferência do $\mathrm{Hg}$ metálico gasoso através de uma bomba de ar $\mathrm{P}_{1}$; conexão com outra bomba $\mathrm{P}_{2}$ que arrasta o vapor de $\mathrm{Hg}$ através de um banho de gelo e retira a umidade, para posterior passagem na célula e detecção do $\mathrm{Hg}$.

\section{Recuperação analítica do $\mathrm{Hg}$}

$\mathrm{Na}$ análise do $\mathrm{Hg}$ por CV-AAS foram preparados padrões compreendidos no intervalo de 0 a $60 \mu \mathrm{g} \mathrm{L}^{-1}$. O padrão de referência certificado utilizado no controle das análises foi o Montana soil SRM-2711, $\mathrm{C}_{\mathrm{Hg} \text { total }}=6,25 \mu \mathrm{g} \mathrm{L}^{-1} \mathrm{e}$ a recuperação analítica do $\mathrm{Hg}$ foi de $94,44 \%$ (desvio padrão $= \pm 0,1313$ ), determinada a partir de 10 medidas de absorbância.

\section{RESULTADOS E DISCUSSÃO}

Com base nas análises macroscópicas dos materiais ao longo dos perfis de solos foi verificado que o perfil TPA, com $161 \mathrm{~cm}$ de profundidade, é representado pelos horizontes $\mathrm{A}_{1}(0-7 \mathrm{~cm}), \mathrm{A}_{2}(7-14$ $\mathrm{cm}), \mathrm{A}_{3}(14-29 \mathrm{~cm}), \mathrm{AB}(29-57 \mathrm{~cm}), \mathrm{BA}(57-89 \mathrm{~cm}), \mathrm{B}_{1}(89-110$ $\mathrm{cm}), \mathrm{B}_{2}(110-135 \mathrm{~cm})$ e $\mathrm{B}_{3}(135-161 \mathrm{~cm})$. O horizonte $A$ apresenta as colorações: preta (10YR 2/1) nos horizonte $\mathrm{A}_{1}$ e $\mathrm{A}_{2}$ e marrom escuro (7,5 YR 3/2) em $A_{3}$. Os horizontes de transição AB e BA apresentam cores variando entre marrom forte (7,5 YR 4/6) a marrom (7,5 YR $4 / 3)$. No horizonte $B$ as colorações variam entre marrom amarelado (10 YR 5/4) a amarelo amarronzado (10YR 6/6). O perfil AD, com $180 \mathrm{~cm}$ de profundidade, apresenta os horizontes $\mathrm{A}_{1}(0-8 \mathrm{~cm}), \mathrm{AB}$ $(8-20 \mathrm{~cm}), \mathrm{BA}(20-59 \mathrm{~cm}), \mathrm{B}_{1}(59-100 \mathrm{~cm}), \mathrm{B} 2(100-162 \mathrm{~cm})$ e $\mathrm{B}_{3}$ $(162-180 \mathrm{~cm})$. O horizonte A é marrom acinzentado escuro (10 YR 4/2) e os outros horizontes são marrom amarelado escuro (10 YR 4/4).

Os dados da análise granulométrica indicaram predominância da fração areia grossa (intervalos em g/100 g nos horizontes superiores dos perfis: 53 a 56 no perfil TPA e 41 a 47 no perfil AD) sobre as demais frações em todos os horizontes dos perfis (intervalos das frações areia fina, silte e argila em g/100 g nos horizontes superiores dos perfis: no perfil TPA 17-19, 19-22 e 6-9 e no perfil AD 17-20, 15-18 e 18-21, respectivamente). Estes dados permitiram identificar que o perfil TPA apresenta textura arenosa nos horizontes $A_{1}$ a BA, textura média (areno-argilosa) no horizonte B e que o perfil AD apresenta textura média em todo o perfil.

Através das análises por DRX foram identificados nos horizontes dos perfis os seguintes minerais: quartzo, caulinita, goethita e anatásio. Estimativas sobre as concentrações destes minerais podem ser obtidas a partir de dados de análises químicas totais do $\mathrm{Si}, \mathrm{Al}$, $\mathrm{Fe}, \mathrm{Ti}$ (Tabelas 1 e 2). Deste modo, as concentrações dos minerais identificados nos perfis TPA e AD podem ser calculadas através das seguintes relações: a da caulinita $\left(\mathrm{Al}_{2} \mathrm{O}_{3} \cdot 2 \mathrm{SiO}_{2} \cdot 2 \mathrm{H}_{2} \mathrm{O}\right)$ pelos teores de $\mathrm{Al}_{2} \mathrm{O}_{3}\left(\mathrm{C}_{\text {caulinita }}=\mathrm{C}_{\mathrm{Al} 2 \mathrm{O} 3} \mathrm{x}\right.$ massa molecular da caulinita $/ \mathrm{m}$.molecular $\mathrm{Al}_{2} \mathrm{O}_{3}$ ); a do quartzo (polimorfo do $\mathrm{SiO}_{2}$ ) a partir da diferença entre $\mathrm{SiO}_{2}$ total e $\mathrm{SiO}_{2}$ contido na caulinita; a da goethita $(\alpha-\mathrm{FeOOH})$ pelos teores de $\mathrm{Fe}_{2} \mathrm{O}_{3}$; a do anatásio (polimorfo de $\mathrm{TiO}_{2}$ ) pelas concentrações de $\mathrm{TiO}_{2}$. De acordo com estas estimativas verificou-se que a caulinita e o quartzo são os minerais mais abundantes em ambos os perfis. Os teores de caulinita aumentam com a profundidade nos dois perfis, sendo mais pronunciado no perfil AD. Goethita, matéria orgânica (MO) e anatásio apresentam concentrações próximas entre si e bem inferiores às concentrações da caulinita e do quartzo em todos os horizontes dos perfis. Observa-se através dos resultados das análises químicas (Tabelas 1 e 2) que os horizontes A do perfil TPA apresentam teores mais altos em $\mathrm{SiO}_{2}$ e matéria orgânica (MO), bem como teores mais baixos em $\mathrm{Al}_{2} \mathrm{O}_{3}$ e $\mathrm{Fe}_{2} \mathrm{O}_{3}$ quando comparados com os horizontes A do perfil AD. A diferença entre os valores de $\mathrm{pH}_{\mathrm{KCl}}$ e $\mathrm{pH}_{\mathrm{H} 2 \mathrm{O}}$ é negativa nos dois perfis, indicando predomínio de cargas negativas nestes solos.

Tabela 1. Componentes principais (\% em peso) e valores de $\mathrm{pH}$ do perfil de solo TPA (fração menor que $2 \mathrm{~mm}$ de diâmetro)

\begin{tabular}{lccccccccc}
\hline Hor. & $\mathrm{Fe}_{2} \mathrm{O}_{3}$ & $\mathrm{Al}_{2} \mathrm{O}_{3}$ & $\mathrm{SiO}_{2}$ & $\mathrm{TiO}_{2}$ & $\mathrm{PF}$ & $\mathrm{MO}$ & $\mathrm{pH}_{\text {aqua }}$ & $\mathrm{pH}_{\mathrm{KCl}}$ & $\Delta \mathrm{pH}$ \\
\hline $\mathrm{A}_{1}$ & 1,85 & 7,38 & 78,36 & 0,55 & 4,78 & 4,28 & 4,5 & 3,83 & $-0,67$ \\
$\mathrm{~A}_{2}$ & 1,96 & 7,57 & 73,71 & 0,77 & 3,70 & 3,16 & 4,9 & 3,89 & $-1,01$ \\
$\mathrm{~A}_{3}$ & 2,21 & 7,91 & 71,85 & 0,99 & 3,60 & 1,93 & 5,11 & 4,18 & $-0,93$ \\
$\mathrm{AB}$ & 2,98 & 9,86 & 70,43 & 1,02 & 1,50 & 0,68 & 5,26 & 4,38 & $-0,88$ \\
$\mathrm{BA}$ & 3,46 & 9,88 & 69,84 & 1,16 & 1,44 & 0,62 & 5,26 & 4,36 & $-0,9$ \\
$\mathrm{~B}_{1}$ & 3,81 & 11,16 & 68,57 & 1,20 & 1,40 & 0,50 & 5,36 & 4,61 & $-0,75$ \\
$\mathrm{~B}_{2}$ & 4,03 & 14,25 & 67,88 & 1,24 & 1,00 & 0,31 & 5,59 & 4,69 & $-0,9$ \\
$\mathrm{~B}_{3}$ & 5,08 & 15,02 & 66,50 & 1,24 & 1,00 & 0,20 & 5,87 & 4,91 & $-0,96$ \\
\hline
\end{tabular}

Tabela 2. Componentes principais (\% em peso) e valores de $\mathrm{pH}$ do perfil de solo $\mathrm{AD}$ (fração menor que $2 \mathrm{~mm}$ de diâmetro)

\begin{tabular}{lccccccccc}
\hline Hor. & $\mathrm{Fe}_{2} \mathrm{O}_{3}$ & $\mathrm{Al}_{2} \mathrm{O}_{3}$ & $\mathrm{SiO}_{2}$ & $\mathrm{TiO}_{2}$ & $\mathrm{PF}$ & $\mathrm{MO}$ & $\mathrm{pH}_{\text {aqua }}$ & $\mathrm{pH}_{\mathrm{KCl}}$ & $\Delta \mathrm{pH}$ \\
\hline $\mathrm{A}_{1}$ & 3,05 & 9,34 & 70,50 & 0,82 & 1,38 & 0,87 & 4,06 & 3,60 & $-0,46$ \\
$\mathrm{AB}$ & 4,58 & 12,46 & 68,13 & 1,46 & 1,82 & 0,81 & 4,13 & 3,73 & $-0,4$ \\
$\mathrm{BA}$ & 5,10 & 15,28 & 65,92 & 2,05 & 2,0 & 0,58 & 4,22 & 4,00 & $-0,22$ \\
$\mathrm{~B}_{1}$ & 5,36 & 16,29 & 65,13 & 2,21 & 3,02 & 0,37 & 4,24 & 4,02 & $-0,22$ \\
$\mathrm{~B}_{2}$ & 6,72 & 17,81 & 63,64 & 2,28 & 2,60 & 0,31 & 4,46 & 4,05 & $-0,41$ \\
$\mathrm{~B}_{3}$ & 7,93 & 19,72 & 63,20 & 2,31 & 3,45 & 0,13 & 4,58 & 4,05 & $-0,53$ \\
\hline
\end{tabular}

As concentrações de mercúrio total nos perfis TPA e AD do sítio Ilha de Terra e de outros sítios arqueológicos da região de Caxiuanã, como os de S. Raimundo e Conceição, ${ }^{20}$ estão indicadas na Tabela 3. Comparando-se as concentrações de $\mathrm{Hg}$ total entre os horizontes dos perfis TPA e AD do sítio Ilha de Terra com as obtidas nos sítios S. Raimundo e Conceição, verifica-se que há 
tendências para o aumento das concentrações de $\mathrm{Hg}$ dos horizontes $\mathrm{A}_{1}$ para os horizontes $\mathrm{B}$, tanto nos perfis TPA e AD, sendo as maiores concentrações verificadas no perfil AD; observa-se que não há diferenças significativas entre as concentrações de $\mathrm{Hg}$ nos horizontes $\mathrm{A}_{1}$ a $\mathrm{B}_{3}$ dos perfis TPA e $\mathrm{AD}$ dos sítios $\mathrm{S}$. Raimundo e Conceição.

Tabela 3. Concentrações de $\mathrm{Hg}_{\text {total }}$ em ng g-1 $\left(\mu \mathrm{kg}^{-1}\right)$ em perfis de solos TPA e $\mathrm{AD}$ (fração menor que $2 \mathrm{~mm}$ de diâmetro) da região de Caxiuanã

\begin{tabular}{lcccccc}
\hline Horizonte & \multicolumn{3}{c}{ Perfis de solos TPA } & \multicolumn{3}{c}{ Perfis de solos AD } \\
& $(\mathrm{a})$ & $\left(\mathrm{b}_{1}\right)$ & $\left(\mathrm{b}_{2}\right)$ & $(\mathrm{a})$ & $\left(\mathrm{b}_{1}\right)$ & $\left(\mathrm{b}_{2}\right)$ \\
\hline $\mathrm{A}_{1}$ & 49 & 188 & 220 & 163 & 230 & 203 \\
$\mathrm{~A}_{2}$ & 52 & 215 & 187 & & & \\
$\mathrm{~A}_{3}$ & 65 & & & & & \\
$\mathrm{AB}$ & 75 & 235 & 237 & 189 & 237 & 199 \\
$\mathrm{BA}$ & 118 & 230 & 252 & 199 & 259 & 229 \\
$\mathrm{~B}_{1}$ & 169 & 257 & 243 & 284 & 289 & 234 \\
$\mathrm{~B}_{2}$ & 198 & 267 & 207 & 297 & 299 & 247 \\
$\mathrm{~B}_{3}$ & 208 & & & 299 & & \\
\hline
\end{tabular}

a) dados obtidos neste estudo; $b_{1}$ e $b_{2}$ ) dados obtidos para os sítios S. Raimundo e Conceição, respectivamente (Carmo, 2008)

Tabela 4. Concentrações sequenciais de $\mathrm{Hg}$ em ng g$^{-1}\left(\mu \mathrm{g} \mathrm{kg}^{-1}\right)$ nas distintas fases dos perfis de solos TPA e AD

\begin{tabular}{lccccccccccc}
\hline & \multicolumn{1}{c}{$\mathrm{C}_{\mathrm{Hg}}\left(\mathrm{ng}_{\mathrm{g}}^{-1}\right)$} & perfil de solo TPA & \multicolumn{6}{c}{$\mathrm{C}_{\mathrm{Hg}}\left(\mathrm{ng}^{-1} \mathrm{~g}^{-1}\right)$} & perfil de solo $\mathrm{AD}$ \\
\hline Hor & 1 & 2 & 3 & 4 & 5 & 1 & 2 & 3 & 4 & 5 \\
$\mathrm{~A}_{1}$ & 27 & 18 & 8 & nd & 49 & 91 & 50 & 19 & nd & 163 \\
$\mathrm{~A}_{2}$ & 30 & 21 & 6 & nd & 52 & & & & & \\
$\mathrm{~A}_{3}$ & 37 & 25 & 4 & nd & 65 & & & & & \\
$\mathrm{AB}$ & 42 & 28 & 3 & nd & 75 & 127 & 65 & 12 & nd & 189 \\
$\mathrm{BA}$ & 76 & 34 & 3 & nd & 118 & 189 & 80 & 12 & nd & 199 \\
$\mathrm{~B}_{1}$ & 114 & 53 & 3 & nd & 169 & 201 & 83 & 6 & nd & 284 \\
$\mathrm{~B}_{2}$ & 133 & 62 & 2 & nd & 198 & 210 & 87 & 6 & nd & 297 \\
$\mathrm{~B}_{3}$ & 138 & 66 & 2 & nd & 208 & 212 & 87 & 2 & nd & 299 \\
\hline
\end{tabular}

1. Hg na fase minerais Fe-Mn; 2. Hg na fase residual; 3. Hg na matéria orgânica (MO), 4. Hg na fase trocável (nd: não detectado), 5. Hg total

Avaliando-se a distribuição das concentrações de matéria orgânica (MO) em relação às de $\mathrm{C}_{\text {total }}$ do mercúrio e dos outros componentes investigados nos dois perfis (Figuras 1-4) verifica-se que a da MO (Figura 1) tende a ser inversa à do $\mathrm{Hg}$ nos dois perfis (Figura 2) e aproxima-se às do alumínio (Figura 3 ) e ferro (Figura 4) em ambos perfis.

Estes dados levam a supor que há correlação positiva e significativa entre $\mathrm{Fe}, \mathrm{Al}$ e $\mathrm{Hg}$ e negativa destes elementos com a $\mathrm{MO}$, sugerindo que o $\mathrm{Hg}$ está intrinsecamente relacionado com os minerais de ferro e de argila, representados nos perfis de solos pela goethita $(\alpha-\mathrm{FeOOH})$ e caulinita $\left(\mathrm{Al}_{2} \mathrm{O}_{3} \cdot 2 \mathrm{SiO}_{2} \cdot 2 \mathrm{H}_{2} \mathrm{O}\right)$, respectivamente. Os tipos de correlações entre o $\mathrm{Hg}$ com $\mathrm{MO}$ e com a goethita e caulinita, indicadas a partir de $\mathrm{C}_{\text {total }}$ de $\mathrm{MO}, \mathrm{Hg}$, $\mathrm{Al}$ e $\mathrm{Fe}$, são compatíveis com os dados analíticos do Hg obtidos através de extrações sequenciais (Tabela 3) sendo que o $\mathrm{Hg}$ foi disponibilizado a partir de cada fase sólida após tratamento com solução extratora adequada, segundo o método empregado, ${ }^{27}$ na seguinte ordem de concentração $\left(\mathrm{ng} \mathrm{g}^{-1}\right)$ nos perfis de solos TPA e $\mathrm{AD}$ : $\mathrm{Hg}-\mathrm{MO}<\mathrm{Hg}$-fase residual $<\mathrm{Hg}$-fase minerais de $\mathrm{Fe}-\mathrm{Mn}$ (Figuras 5 e 6, respectivamente). Correlações positivas do $\mathrm{Hg}$ com Fe e Al foram também verificadas nos horizontes superficiais dos solos da região do Tapajós-Pará e a interpretação dada para este aspecto é que os minerais de $\mathrm{Fe}$ e $\mathrm{Al}$ nesta região, representados por oxi-hidróxidos de $\mathrm{Fe}$ e Al, controlam a poluição ambiental do Hg nos sistemas aquáticos. ${ }^{9}$

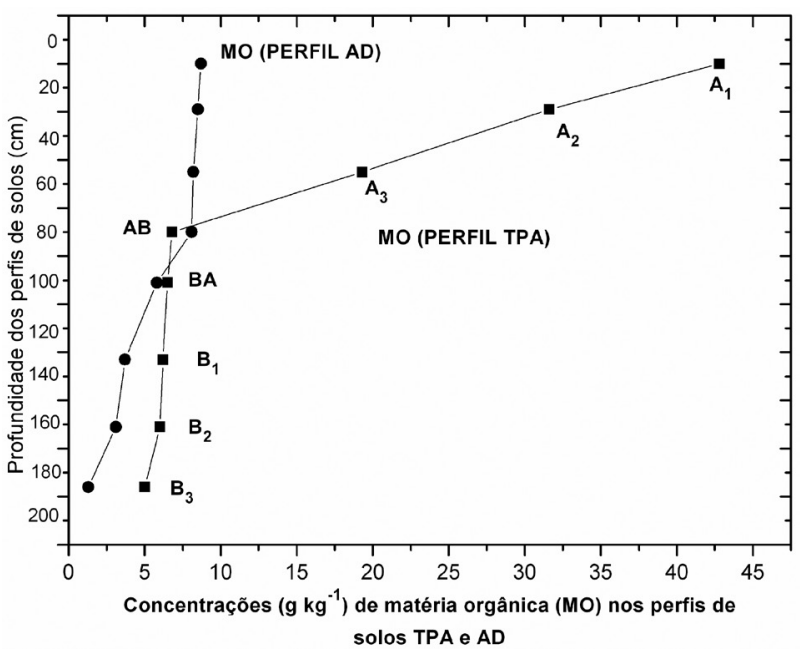

Figura 1. Distribuição da concentração de carbono orgânico total expressa como matéria orgânica (MO) nos perfis de solos com terra preta arqueológica (perfil TPA) e solos de área adjacente (perfil $A D$ )

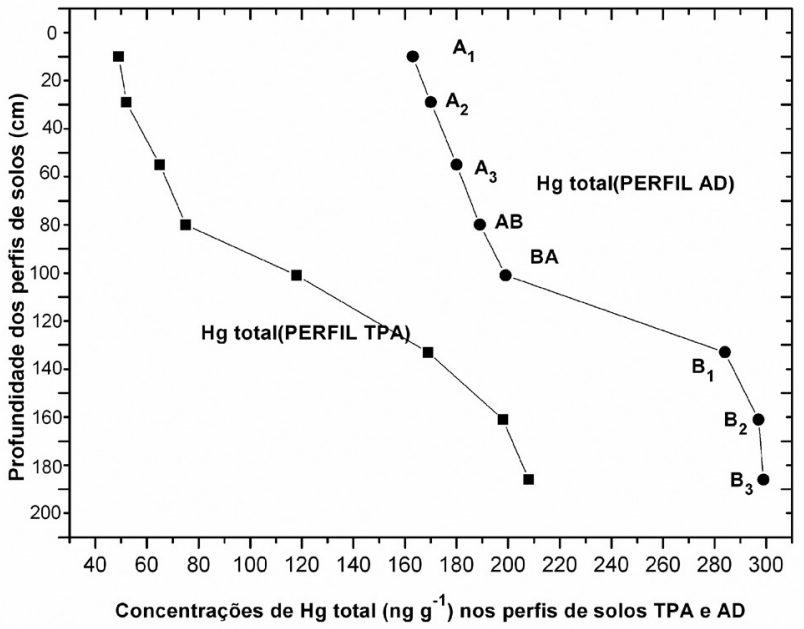

Figura 2. Distribuição da concentração total de Hg nos perfis de solos com terra preta arqueológica (perfil TPA) e solos de área adjacente (perfil AD)

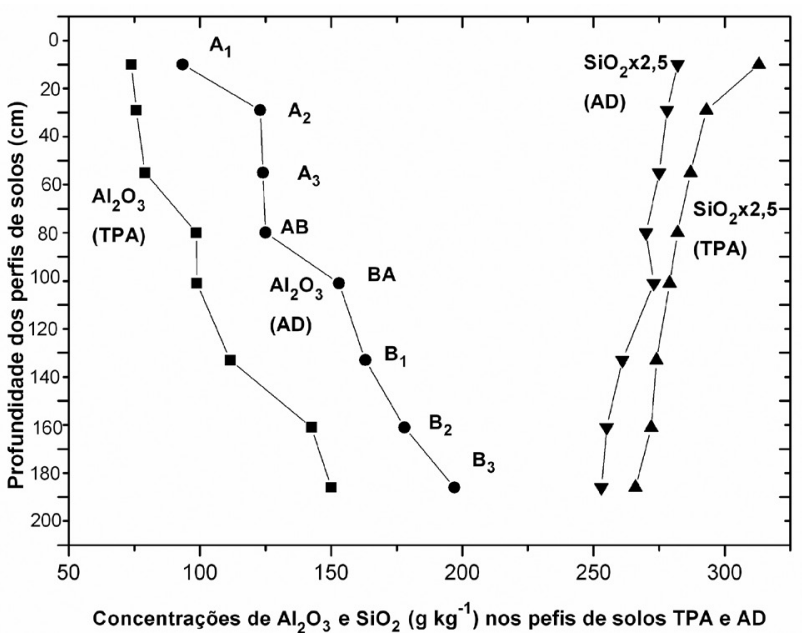

Figura 3. Distribuição da concentração total do $\mathrm{Al}$ ( $\mathrm{como}_{2} \mathrm{O}_{3}$ ) e Si (como $\mathrm{SiO}_{2}$ ) nos perfis de solos com terra preta arqueológica (perfil TPA) e solos de área adjacente (perfil $A D$ ) 


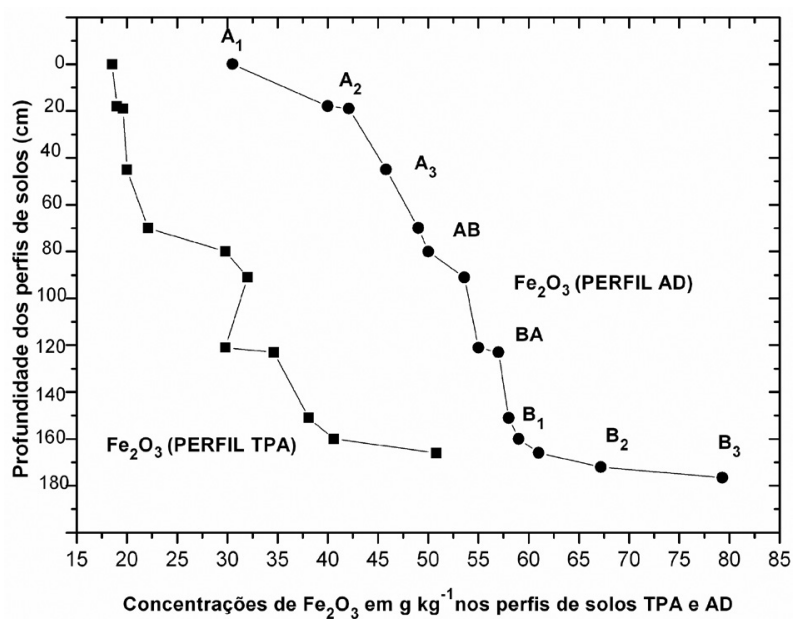

Figura 4. Distribuição da concentração total do Fe expressa como $\mathrm{Fe}_{2} \mathrm{O}_{3}$, nos perfis de solos com terra preta arqueológica (perfil TPA) e solos de área adjacente (perfil $A D$ )

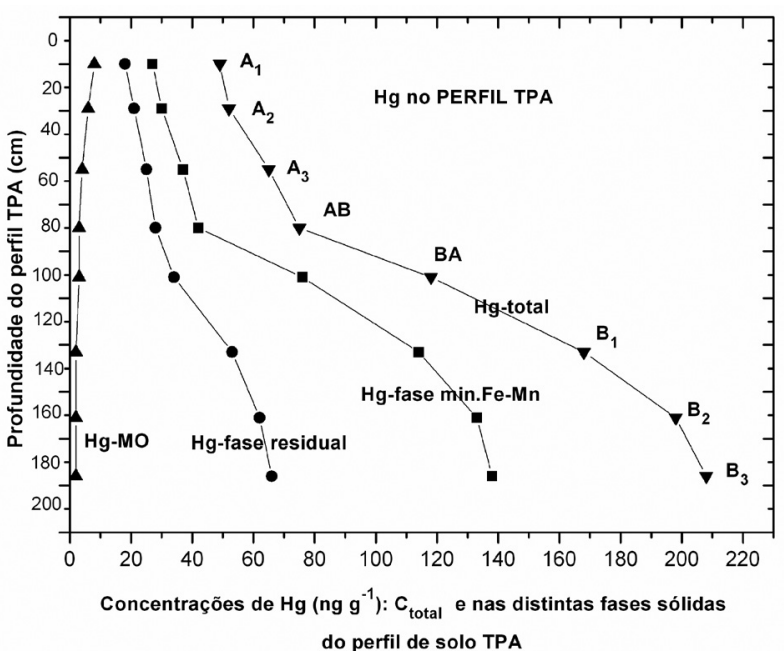

Figura 5. Concentrações de Hg disponibilizadas nas fases correspondentes à matéria orgânica, minerais de Fe e Mn e fase residual (minerais de argila) nos solos com terra preta arqueológica (perfil TPA)

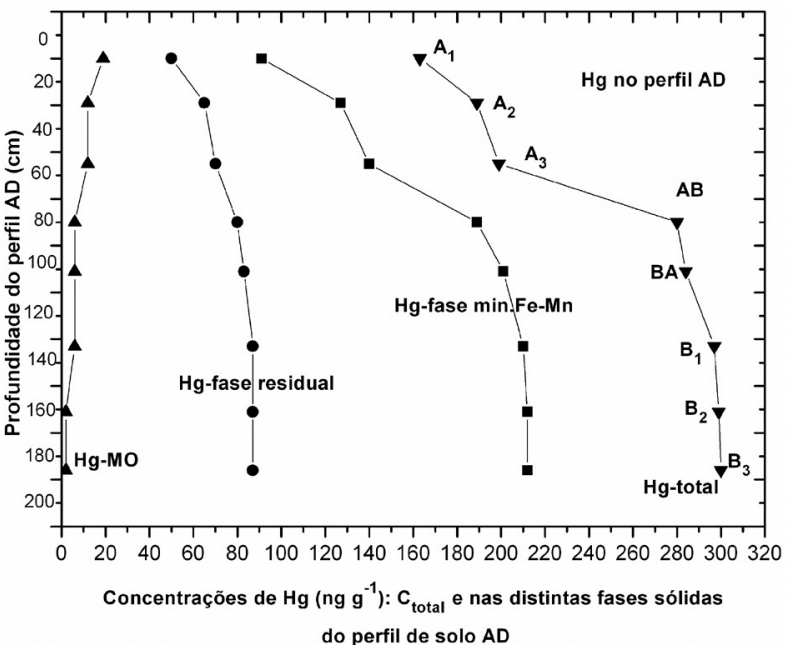

Figura 6. Concentrações de $\mathrm{Hg}$ disponibilizadas nas fases correspondentes à matéria orgânica, minerais de Fe e Mn e fase residual (minerais de argila) nos solos de área adjacente (perfil $A D$ )

\section{CONCLUSÕES}

Os aspectos texturais e as principais características químicas e mineralógicas dos perfis de solos TPA e AD do sítio arqueológico de Ilha de Terra enquadram-se nos dados obtidos em outros sítios da região de Caxiuanã e, ao mesmo tempo, mostram afinidades entre si, indicando que os solos TPA se desenvolveram sobre os Latossolos Amarelos pré-existentes. Por sua vez estes derivaram da alteração de antigos perfis lateríticos. Antes da ocupação humana os solos desses perfis deveriam constituir uma única unidade de solo, mas as atividades desenvolvidas pelos antigos habitantes da região durante longo período de tempo provocaram mudanças nos horizontes superiores desta unidade de solo, que foram evidenciados neste estudo através dos seguintes dados: 1 . mudança da cor amarela dos Latossolos para as cores preta, marrom escuro e marrom claro, devido ao acúmulo de matéria orgânica originada de resíduos animais e vegetais; pela alteração da textura média (areno-argilosa) dos latossolos para arenosa nos solos com TPA devido ao assentamento humano que propiciou o decréscimo dos teores de $\mathrm{Al}$ e aumento nos de Si (Figura 3) devido à formação do quartzo; 2. decréscimo nos teores de Fe, devido à solubilização parcial da goethita que deve ter ocorrido a partir da reação de oxi-redução entre o $\mathrm{Fe}^{3+}$ da goethita e a matéria orgânica em presença de micro-organismos que aceleram a velocidade desta reação $;^{37}$ 3. decréscimo dos teores de $\mathrm{Hg}$, devido especialmente à solubilização da goethita nos solos com TPA da região de Caxiuanã.

Nos solos dessa região é muito provável que as concentrações de $\mathrm{Hg}$ sejam controladas pelas da goethita e caulinita. Nos horizontes superiores dos solos com TPA as condições de Eh-pH são favoráveis à solubilização parcial da goethita e desestabilização da caulinita e consequente liberação do $\mathrm{Hg}$ previamente contido em posições adequadas nas estruturas desses minerais. Esta hipótese é reforçada por dados experimentais que indicam a ligação do $\mathrm{Hg}$ nos óxidos e hidróxidos de ferro e nos grupos silanois da caulinita. ${ }^{38,39} \mathrm{O} \mathrm{Hg}$ liberado não ocorre como íon $\mathrm{Hg}^{2+}$ livre, mas complexado como hidróxido, formando distintos íons complexos de acordo com as condições de $\mathrm{pH}$, como o $\mathrm{HgOH}^{+}$que é o íon complexo mais estável em pH ácido, ${ }^{40}$ típico da região de Caxiuanã. O mercúrio é mobilizado como $\mathrm{HgOH}^{+}$em direção aos horizontes mais profundos dos solos e é novamente ligado à goethita e à caulinita.

\section{MATERIAL SUPLEMENTAR}

A Figura 1S, disponível em http://quimicanova.sbq.org.br na forma de arquivo .PDF com acesso livre, mostra a localização de vários sítios com terra preta arqueológica (TPA) às margens da baía de Caxiuanã - Pará.

\section{AGRADECIMENTOS}

Ao Conselho Nacional de Desenvolvimento Científico e Tecnológico $(\mathrm{CNPq})$ pelo apoio financeiro (Projeto assinaturas geoquímicas de terra preta arqueológica) e concessão de bolsas de IC (W. T. S. dos Santos) e produtividade em pesquisa - PQ (M. L. da Costa, V. P. Lemos e K. das G. F. Dantas).

\section{REFERÊNCIAS}

1. Wedepohl, K. H.; Geochim. Cosmochim. Acta 1995, 59, 1217.

2. Reiman, C.; Caritat, P.; Chemical Elements in the Environment: Factsheets for the Geochemist and Environment Science Technology, Spring Verlag Berlin: New York, 1998.

3. Lacerda, L. D.; Nature 1995, 374, 20.

4. Malm, O.; Environ. Res. 1998, 77, 73.

5. Veiga, M. M.; Meech, J. A.; Onate, N.; Nature 1999, 368, 816 
6. Roulet, M.; Lucotte, M.; Water, Air, Soil Pollut. 1995, 80, 1079.

7. Roulet, M.; Lucotte, M.; Canuel, R.; Rheault, I.; Tran, S.; De Freitos Gogh, Y. G.; Farella, N.; Souza do Valle, R.; Sousa Passos, C. J.; Jesus da Silva, E.; Mergler, D.; Amorim, M.; Sci. Total Environ. 1998b, 213, 203.

8. Roulet, M.; Lucotte, M.; Farella, N.; Serique, G.; Coelho, H.; Sousa Passos, C. J.; Silva, E. J.; Andrade, P. S.; Mergler, D.; Guimarães, J. R. D.; Amorim, M.; Water, Air, Soil Pollut. 1999, 112, 297.

9. Roulet, M.; Lucotte, M.; Saint-Albin, A.; Tran, S.; Rhéault, I.;Farella, N.; Jesus da Silva, E.; Dezencourt, J.; Souza Passos, C. J.; Santos Soares, G.; Guimarães, J. R. D.; Mergler, D.; Amorim, M.; Sci. Total Environ. 1998, 223, 1.

10. Brabo, E. S.; Angélica, R. S.; Silva, A.; Fayal, K. R. K.; Mascarenhas, A. F. S.; Santos, E. C. O.; Jesus, I. M.; Loureiro, E. C. B.; Water, Air, Soil Pollut. 2003, 147, 61.

11. Brabo, E. S.; Tese de Doutorado, Universidade Federal do Pará, Brasil, 2006.

12. Oliveira, S. M. B.; Melfi, A. J.; Fostier, A. C.; Favero, D. I. T.; Boulet, R.; Water, Air, Soil Pollut. 2001, 26, 1.

13. Oliveira, S. M. B.; Larizzatti, J. H.; Favero, D. I. T.; Geochim. Brasiliensis 2002, 16, 23.

14. Costa, M. L.; Angélica, R. S.; Costa, N. C.; J. Geochem. Explor. 1999, $67,33$.

15. Kern, D. C.; Tese de Doutorado, Universidade Federal do Pará, Brasil, 1996.

16. Costa, M. L.; Kern, D. C.; J. Geochem. Explor. 1999, 66, 369.

17. Lisboa, P. L. B.; Estação Científica Ferreira Pena/ECFPn-Caxiuanã, Museu Paraense Emílio Goeldi: Belém, 1997.

18. Smith, N. J. H.; Annals of the Assoc. Am. Geographers 1980, 70, 553.

19. Neves, E. G.; Petersen, J. B.; Bartone, R. N.; Silva, C. A. Em Historical and socio-cultural origins of Amazonian Dark Earths; Lehmann, J.; Kern, D. C.; Glaser, B.; Woods, W. I.; eds.; Kluwer Academic Publishers: Dordrecht, 2003.

20. Carmo, M. S.; Tese de Doutorado, Universidade Federal do Pará, Brasil, 2008 .

21. Lemos, A.; Santos, P. B.; Manual de Descrição e Coleta de Solos em Campo, SBCS/SNLCS: Campinas-SP, 1996.
22. Munsell Collors Company; Munsell soil colors charts, Baltimore, 2000.

23. Empresa Brasileira de Pesquisa Agropecuária; Manual de métodos de análise de solo, $2^{\text {a }}$ ed., EMBRAPA, CNPS: Rio de Janeiro, 1997.

24. USDA; Soil Survey Laboratory Methods Manual. Soil Survey Investigations Report, Version 3.0, Washington, USDA of Communications, 1996.

25. Bennett, H.; Reed, R. A.; Chemical Methods of Silicate Analysis, Academic Press: New York, 1971.

26. Akagi, H.; Nishimura, H. Em Especiation of Mercury in the Environment; Suzuki, T.; Imura, N.; Clarkson, T. W.; eds.; Advance in Mercury Toxicology: New York, 1996.

27. Tessier, A.; Campbell, P. G. C.; Bisson, M.; Anal. Chem. 1979, 51, 844.

28. Chao, T. T.; Zhou, L.; J. Soil Sci. Soc. Am. Proc. 1983, 47, 225.

29. Miller, W. P.; Martens, D. C.; Zelanzny, L. W.; Soil Sci. Am. J. 1986, 50, 598.

30. Keller, C.; Verdy, J. C.; J. Environ. Qual. 1994, 23, 987.

31. Hall, G. E. M.; Vaive, J. E.; Beer, R.; Hoashi, M.; J. Geochem. Explor. 1996, 56, 59 .

32. Lã, O. R.; Barra, C. M.; Amaral Sobrinho, N. M. B.; Mazur, N.; Velloso, A. C. X.; Quim. Nova 2003, 26, 323.

33. Templeton, D. M.; Ariese, F.; Cornelis, R.; Danielsson, L. G.; Muntau, H.; van Leeuwen, H. P.; Lobinski, R.; Pure Appl. Chem. 2000, 72, 1453.

34. Davidson, C. M.; Duncan, A. L.; Littlejohn, A. M. U.; Garden, L. M.; Anal. Chim. Acta 1998, 363, 45.

35. Micaroni, R. C. C. M.; Bueno, M. I. M. S.; Jardim, W. F.; Quim. Nova 2000, 23, 487.

36. Akagi, H.; Malm, O.; Branches, F. J. P.; Environ. Sci. Technol. 1996, 4, 199.

37. Lovley, D. R.; Woodward, J. C.; Chem. Geol. 1996, 132, 19.

38. Bonnissel, G. P.; Alnot, M.; Lickes, J. P.; Ehrhardt, J. J.; Behra, P.; J. Colloid Interface Sci. 1999, 215, 313.

39. Singh, J.; Huang, P. M.; Hammer, U. T.; Liaw, W. K.; Clays Clay Miner. 1996, $44,41$.

40. Navarro, A.; Biester, H.; Mendonza, J. L.; Cordellach, E.; Environ. Geol. 2006, 49, 1089 


\section{COMPORTAMENTO DO MERCÚRIO EM PERFIS DE SOLOS DO SÍTIO ILHA DE TERRA-CAXIUANÃ, PARÁ}

Robson da Silva Gurjão, Vanda Porpino Lemos*, Marcondes Lima da Costa, Heronides Adonias Dantas Filho, Kelly das Graças Fernandes Dantas e Wivian Thais dos Santos Lima

Instituto de Ciências Exatas e Naturais, Universidade Federal do Pará, 66075-780 Belém - PA, Brasil

Dirse Clara Kern

Museu Paraense Emílio Goeldi, Belém - PA, Brasil

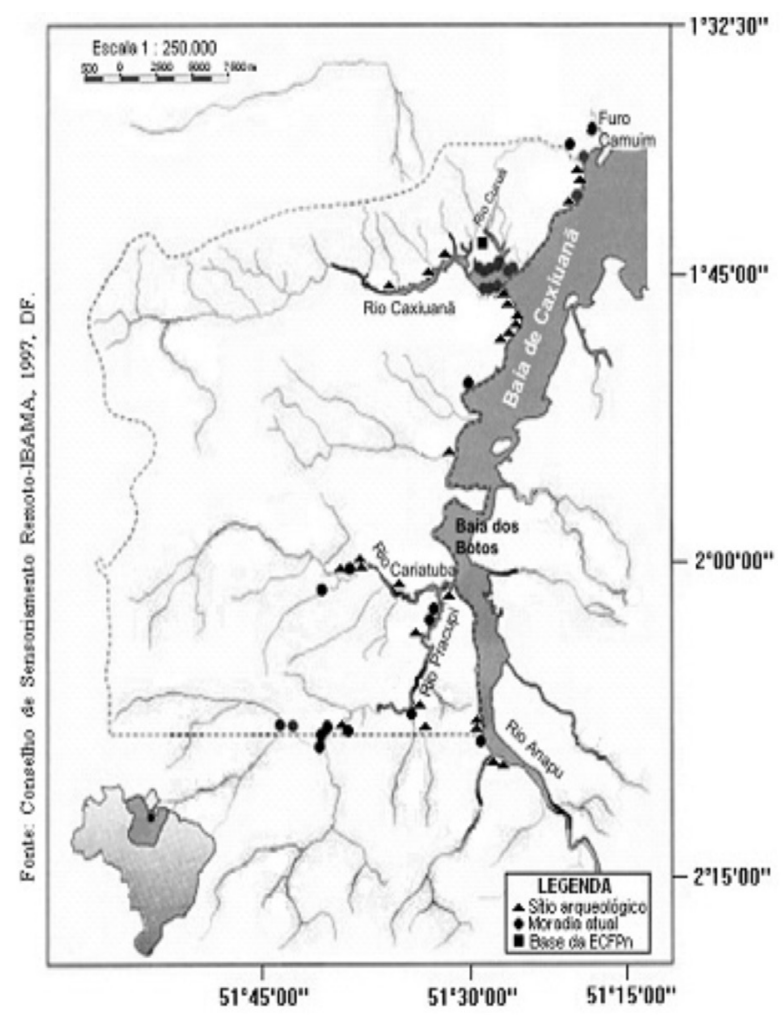

Figura 1S. Mapa de localização da região de Caxiuanã, indicando a base da Estação Científica Ferreira Pena (ECFP) e vários sítios arqueológicos às margens da Baía de Caxiuanã (o sítio arqueológico Ilha de Terra está situado no furo Camuim, extremo norte da Baía de Caxiuanã) 